Cannabis-related paranoia

\title{
Cognitive mechanisms in cannabis-related paranoia; \\ Initial testing and model proposal
}

\begin{abstract}
Cannabis-use increases severity of symptoms and risk of relapse for people with psychosis. Childhood sexual abuse and high schizotypy increase the risk further. The mechanisms involved remain unclear, and this limits psychological therapies. In three linked studies, we examined the role of two candidate mechanisms - external attribution and cognitive fusion. Study 1 examined these processes in a general population sample and showed that paranoia, psychotic-type experiences, and linked distress were higher in cannabis-users, and mediated by cognitive fusion but not external attribution. Study 2 examined the impact of established risk factors in general population cannabis-users and showed that external attribution and cognitive fusion partially or fully accounted for the effects of childhood sexual abuse and schizotypy on paranoia, psychotic-type experiences and linked distress. Study 3 examined these same processes in a clinical population of people with psychosis and found that external attribution and cognitive fusion partially or fully accounted for the impact of gender, age of first use, sexual abuse and schizotypy. External attribution and cognitive fusion may be key mechanisms in the maintenance of cannabis-related paranoia and account for the impact of established risk factors. We present a cognitive model incorporating these processes to inform clinical practice.
\end{abstract}


Cannabis-related paranoia

\section{Introduction}

\section{The relationship between cannabis and psychosis}

Longitudinal general population studies suggest that cannabis-use predicts later psychotic experiences (Gage, Hickman, \& Zammit, 2016), and earlier and heavier use both increase risk (Marconi, Di Forti, Lewis, Murray, \& Vassos, 2016; Mustonen et al., 2018). While epidemiological studies suggest the relationship may be bidirectional (Ferdinand et al., 2005; Griffith-Lendering et al., 2013), longitudinal research shows that the effects of cannabis on psychosis remain when reverse causality is taken into account (Murray, Quigley, Quattrone, Englund, \& Di Forti, 2016; Radhakrishnan, Wilkinson \& D'Souza, 2014). The weight of evidence now clearly indicates that heavy cannabis-use increases risk of psychosis (Colizzi \& Murray, 2018).

In people with a diagnosis of schizophrenia, cannabis-use increases symptom severity (Katz, Durst, Shufman, Bar-Hamburger, \& Grunhaus, 2010) and likelihood of relapse (Manrique-Garcia et al., 2014; van der Meer \& Velthorst, 2015). In those with first episode psychosis (FEP), cannabis-use predicts symptom severity five years later (Clausen et al., 2013), and people at "ultra-high risk" are more than four times as likely to develop psychosis if using cannabis (McHugh et al., 2017). A metaanalysis of outcomes in people with psychosis who continue or stop cannabis found that ongoing use increases symptom severity, impaired functioning, risk of relapse and duration of admissions (Schoeler et al., 2016).

The reasons why people use cannabis are less clear. Self-medication hypotheses suggest that people use substances to regulate painful affect (Khantzian 1997), including those with psychosis who use cannabis to manage dysphoria (Gregg, Barrowclough, \& Haddock, 2007), social contact and psychotic experiences (Mane et al., 2015; Green, Kavanagh, \& Young, 2004). However, there is little empirical support for these hypotheses (Gregg, et al., 2007; Lembke 2012), leading some to suggest that people with psychosis use cannabis for the same reasons as the general population - for intoxication and relaxation effects (Kolliakou, Joseph, Ismail, Atakan, \& Murray, 2011). 
Cannabis-related paranoia

Overall, there is broad agreement that cannabis-use does not necessarily cause psychosis but increases likelihood of psychotic-type experiences such as paranoia, in vulnerable groups (Ksir \& Hart, 2016), and that we need to examine the impact of cannabis in people at higher risk of psychosis in order to develop effective therapies (Gage et al., 2016).

\section{Psychosocial risk factors for cannabis-related psychosis}

A number of factors increase risk of psychosis associated with cannabis-use, including male gender (Hamilton, Galdas, \& Essex, 2015), childhood sexual abuse (CSA) (Harley et al., 2010; Konings et al., 2012; Shevlin, Murphy, Houston, \& Adamson, 2009), schizotypy (Horan, Blanchard, Clark, \& Green, 2008) and younger age at first use (Day, Goldschmidt, Day, Larkby, \& Richardson, 2014; Valmaggia et al., 2014).

Early adversity increases risk of cannabis-use (Khoury, Tang, Bradley, Cubells, \& Ressler, 2010) and psychosis (Varese et al., 2012), and is more prevalent still for those with both (Harley et al., 2010). The risk posed by cannabis may be stronger for people who have experienced early abuse (Konings et al., 2012); indeed, Shevlin et al. (2009) found that cannabis increased risk of psychosis only in those with a sexual abuse history.

Unsurprisingly, schizotypy also increases risk of psychosis (Horan et al., 2008). Schizotypy ranges from common dream-like states to odd perceptual experiences and disorganized thinking. Cannabis-users tend to have high levels of schizotypy (Fridberg, Vollmer, O'Donnell, \& Skosnik, 2011), and people with high schizotypy report more psychotic experiences during and after intoxication (Barkus \& Lewis, 2008; Mason et al., 2009).

While the relationship between cannabis-use and psychotic-type experiences is now well established, the psychological mechanisms and interactions with psychosocial risk factors remain unclear. This limits our therapeutic options. External attribution and cognitive fusion are possible but untested processes contributing to the maintenance of distress in cannabis-related paranoia. 
Cannabis-related paranoia

\section{External attribution}

People with clinical levels of paranoia are more likely than depressed and non-clinical groups to make external personal attributions, i.e. blame others for ambiguous events. For people vulnerable to paranoia, this bias may result in misattribution of intoxication effects, particularly in the context of heightened anxiety (which predisposes threat-based appraisals). For example, wariness of others may be interpreted as evidence that others are intending to cause us harm, rather than a common effect of cannabis intoxication. In an experimental study of THC in people with non-clinical paranoia, informing participants of the likely effects of the drug did not mitigate subsequent paranoia (Freeman et al., 2014). Freeman and colleagues concluded that cannabis causes paranoia through increased negative affect and anomalous experiences. Alternatively, it may be that information available when not intoxicated is not accessed when intoxicated due to the activation of this bias. Richardson, Hughes, Leech, and Raman (2014) suggest that external attribution may affect the relationship between cannabis-use and psychotic experiences, but this remains untested.

\section{Cognitive fusion}

Cognitive fusion is a meta-cognitive process involving "excessive attachment to the literal content of human thought" (Strosahl, Hayes, Wilson \& Gifford, 2004, p.32) such that cognition dominates experience and behaviour (Bolderston et al., 2019). Cognitive fusion is a key process in the Acceptance and Commitment Therapy (ACT) transdiagnostic model of psychopathology. Cognitive theory identifies the broader but overlapping process of decentering - the inability to "step back" from compelling beliefs and perceptions, which is identified as a key feature of psychopathology crossdiagnostically (Bernstein et al., 2015). When thoughts, images and other internal events are experienced as necessarily accurate reflections of self or reality, we are vulnerable to patterns of thinking, feeling and behaving associated with recurrent ill-health (Teasdale et al., 2002). 
Cannabis-related paranoia

In a qualitative study of threat experiences, people with a diagnosis of schizophrenia characterised by paranoia described being "trapped in thinking" and unable to distance themselves from their threat beliefs (Newman-Taylor \& Stopa, 2013). Similarly, "believability" (a proxy for cognitive fusion) of psychotic experiences mediated rehospitalisation following ACT for in-patients diagnosed with psychosis (Bach, Hayes, \& Gallop, 2012). It is likely that intoxication further impairs the ability to defuse from transient internal experience, though the role of cognitive fusion in this context is untested.

\section{Current studies}

We examined the role of candidate psychological mechanisms in the relationship between cannabis and other established risk factors for psychosis, with psychotic experiences in general population, cannabis-using and clinical samples. We predicted that:

1. Study 1: In a general population sample, external attribution and cognitive fusion will account for the relationship between cannabis-use and higher levels of (i) current paranoia, (ii) psychotic-type experiences more broadly, and (iii) distress.

2. Study 2: In a cannabis-using sub-sample, external attribution and cognitive fusion will account for the relationship between established risk factors for cannabis-related psychosis (male gender, CSA, earlier age of first use and schizotypy) and higher levels of (i) current paranoia, (ii) psychotictype experiences and (iii) distress.

3. Study 3: In a sample of people with psychosis, (i) those with a history of cannabis use will have higher levels of paranoia, psychotic experiences, and distress, and this will be accounted for by external attribution and cognitive fusion, (ii) in those with a history of cannabis-use, risk factors (male gender, CSA, younger age of first use and schizotypy) will be associated with paranoia, psychotic experiences and distress, and this will be accounted for by external attribution and cognitive fusion, and (iii) use of cannabis to manage negative affect will be associated with higher 
Cannabis-related paranoia

levels of paranoia, psychotic experiences and distress, and this will be accounted for by external attribution and cognitive fusion.

\section{Materials and methods}

Ethical approval for Studies 1 and 2 was given by University of -, (reference 14594/17075/23281). Ethical approval for Study 3 was given by University of -, (reference 23775), and - NHS Trust Research Ethics Committee (reference 16/LO/1830).

\section{Design}

All three studies used a cross-sectional design.

\section{Participants and procedure (Study 1)}

An opportunity sample $(n=296)$ was recruited via university, public-access research and crowd sourcing websites. Participants were $>=16$ years, able to read English, and had either used cannabis in the past three months $(n=181,61.1 \%)$ or had never used cannabis $(n=115,38.9 \%)$. Those who had used cannabis but not in the past three months were not included in this analysis but were included in Study 2. Ages ranged from $16-75(\mathrm{M}=26.45, S D=11.2)$ years. The majority identified as female $(n=191,65.0 \%)$ and of White ethnicity $(n=233 ; 78.7 \%)$.

Participants gave informed consent and completed demographic and cannabis-use items, the Paranoia Scale (Fenigstein \& Vanable, 1992), Prodromal Questionnaire-Brief (Loewy, Pearson, Vinogradov, Bearden, \& Cannon, 2011) and Cognitive Fusion Questionnaire (Gillanders et al., 2014).

\section{Participants and procedure (Study 2)}


Cannabis-related paranoia

Study 2 participants constituted a sub-sample $(n=172)$ of those screened for Study 1 , who had used cannabis in the past year. Most had first used cannabis $>=16$ years $(62.8 \% ; n=108)$, and $37.2 \%(n=64)$ had first used before 16 years. Participants answered additional questions regarding risk factors for cannabis-related paranoia, specifically CSA (using the Childhood Sexual Trauma Questionnaire; Houston, Murphy, Shevlin, \& Adamson, 2011) and schizotypy (using the Schizotypal Personality Questionnaire-Brief Revised Updated; Davidson, Hoffman, \& Spaulding, 2016).

\section{Participants and procedure (Study 3)}

The clinical sample $(n=60)$ was recruited from community mental health services in southern England. Participants were $>=16$ years. Approximately two thirds were male $(n=38 ; 63.3 \%)$ and a third female $(n=22 ; 36.7 \%)$. Ages ranged from 17-69 $(M=39.73, S D=13.06)$ years. The majority identified as of White ethnicity $(n=53 ; 88.3 \%)$. Participants had received current diagnoses of schizophrenia ( $n=43$; $71.7 \%)$, FEP ( $n=11 ; 18.3 \%)$, schizoaffective disorder $(n=4 ; 6.7 \%)$, post-natal psychosis $(n=1 ; 1.7 \%)$ and bipolar disorder with psychotic symptoms $(n=1 ; 1.7 \%)$. The majority had used cannabis $(n=38$; $63.3 \%)$ and a minority $(n=4 ; 6.7 \%)$ had used in the past three months. Participants gave informed consent and completed demographic and cannabis-use items, the Paranoia Scale (Fenigstein \& Vanable, 1992), Prodromal Questionnaire-Brief Version (Loewy et al., 2011), Cognitive Fusion Questionnaire (Gillanders et al., 2014) and Childhood Sexual Trauma Questionnaire (Houston et al., 2011). Study 3 participants were also asked whether they used cannabis to self-medicate.

\section{Measures}

Paranoia Scale (PS; Fenigstein \& Vanable 1992). The PS is a 20-item measure of paranoid thinking in the general population. Items are rated on a 5-point scale yielding a total paranoia score. The PS has good internal consistency $(\alpha=.84)$ and test-retest reliability $(\alpha=.70)$. Internal consistency was 
Cannabis-related paranoia

excellent for the current general population sample $(\alpha=.94)$ and acceptable for the clinical sample $(\alpha=.77)$.

Prodromal Questionnaire-Brief Version (PQB; Loewy et al., 2011). This is a 21-item measure of psychotic-type experiences. Participants indicate the presence/absence of prodromal experiences and linked distress on a 5-point scale, resulting in prodromal experiences (PBQ-experience) and distress (PBQ-distress) sub-scores. For the purpose of this study we made three changes: (1) participants were asked about their experiences over the previous three months (not one month), (2) we removed the statement asking respondents to exclude experiences under the influence of substances, and (3) we included causal attribution options from the IPSAQ (Kinderman \& Bentall, 1996) plus an additional option being under the influence of drugs or alcohol, for example:

Have you had experiences with telepathy, psychic forces, or fortune telling?

\section{$\square$ YES $\square$ NO If YES: What do you think caused this?}

a. Something about you

b. Something about someone else

c. Something about the situation (circumstances or chance)

d.Being under the influence of drugs or alcohol

Experiences attributed to $\mathrm{b}$ or $\mathrm{c}$ were totalled to give an external attribution score. Internal consistency for the current general population sample was good for prodromal experiences $(\alpha=.83)$ and distress $(\alpha=.84)$. Internal consistency for the clinical sample was acceptable for prodromal experiences $(\alpha=.79)$ and excellent for distress $(\alpha=.91)$.

Cognitive Fusion Questionnaire (CFQ; Gillanders et al., 2014). The CFQ is a 7-item measure of the extent to which an individual becomes fused with the literal content of their thoughts. Respondents rate items on a 7-point scale yielding a total score. The CFQ has excellent internal consistency $(\alpha=.90)$ 
Cannabis-related paranoia

and good test-retest reliability $(r=.80)$. Internal consistency was excellent for both the current general population $(\alpha=.94)$ and clinical $(\alpha=.97)$ samples.

Childhood Sexual Trauma Questionnaire (ST; Houston et al., 2011). The ST comprises six questions regarding sexual trauma before and after 16 years. We used the three items prior to the age of 16 , for example: Before the age of 16, did anyone have sexual intercourse with you without your consent? Participants respond yes, no, or don't know/does not apply. Any of the three questions answered yes were classed as CSA present.

Schizotypal Personality Questionnaire-Brief Revised Updated (SPQ-BRU; Davidson et al., 2016). This is a 32-item measure of schizotypy. Participants rate items on a 5-point scale, yielding a total score. The original scale does not report internal consistency. Internal consistency was excellent for the current general population sample $(\alpha=0.92)$ and $\operatorname{good}(\alpha=.86)$ for the clinical sample.

Cannabis use items. Participants were asked to state whether they had used cannabis and if so, age of first use, and whether they had used in the past three months. Clinical participants were also asked "Do/did you ever use cannabis to cope with problems such as depression, anxiety or paranoia?" (response options: a lot/quite a lot/sometimes/never) to assess use of cannabis for self-medication.

\section{Data analysis}

We used SPSS version 24 to inspect distribution of data, confirm normality and calculate correlations. Variables for Studies 1 and 2 were normally distributed except for external attribution, which was kurtotic in the whole sample and in the group of current cannabis-users. Variables for Study 3 were normally distributed except for external attribution; many participants scored 0, but kurtosis was -.67 and skewness .80 so the scale was deemed normally distributed.

We used PROCESS for SPSS (Hayes, 2017) to complete mediation analyses. It has become common practice to use kappa-squared to assess indirect effect sizes, but problems have been identified 
Cannabis-related paranoia

with this (Wen \& Fan, 2015) and there is debate about the merits of other effect size measures and ease of interpretation (Field, 2014; Hayes, 2017). Following Field (2014), we report the standardized $b$ statistic for the indirect effect and the Sobel test.

Study 1 examined whether external attribution and cognitive fusion mediated the relationships between cannabis use and each of paranoia, psychotic-type experiences and distress. The independent variable was cannabis-use (never used/used in the past three months). The dependent variables were paranoia (PS), psychotic-type experiences (PBQ-experience) and distress (PBQ-distress). Mediating variables were external attribution (IPSAQ-external attribution) and cognitive fusion (CFQ).

Study 2 assessed the role of external attribution and cognitive fusion in the relationship between established risk factors for cannabis-related paranoia and paranoia, psychotic-type experiences and distress, in people who had used cannabis in the past 12 months, using MANOVA/MANCOVA and mediation. The independent variables were gender (male/female), presence of childhood sexual abuse (ST), age of first cannabis-use (under 16/16 and above), and schizotypy (SPQ-BRU). The dependent variables and mediators were as for Study 1.

For Study 3 the sample size was insufficient for regression or mediation analyses when split by cannabis-use, so we used MANCOVAs. The analysis requiring the largest sample size included three dependent variables - measures of paranoia (PS), psychotic symptoms more broadly (PQBexperience) and linked distress (PBQ-distress). G-power calculations suggested a sample size of 44 for a medium effect size with two levels of the independent variable. The total sample size of 60 was sufficient to compare those with and without a cannabis-use history. The sub-sample of people who had used cannabis $(n=38)$ was under the recommended size, so other independent variables were analysed using MANOVAs: gender (male/female), history of CSA (yes/no), age of first use (under 16/16 and above), schizotypy (low/high), and using cannabis to cope with negative affect (yes/no). 
Cannabis-related paranoia

\section{Results}

Study 1: Do external attribution and cognitive fusion mediate the relationships between cannabis use and each of paranoia, psychotic-type experiences and distress?

\section{Correlations}

Table 1 gives descriptive statistics and Pearson's correlation coefficients. Paranoia, psychotic-type experiences, distress, external attribution and cognitive fusion correlated in both recent users and nonusers. Associations were generally medium to large in size. The exception was the very large relationship between psychotic-type experiences and distress $(r=.93-.95)$.

*Table 1 about here*

\section{Mediation analyses ${ }^{1}$}

External attribution did not mediate the relationships between cannabis use and paranoia $(b=-.08, \mathrm{BCa}$ $\mathrm{CI}[-1.36, .1 .23])$, psychotic-type experience $(b=-.03$, BCa CI[-.60,.51]) or distress $(b=-.10, \mathrm{BCa} \mathrm{CI}[-$ $1.72, .1 .52])$. Cognitive fusion mediated the relationship between cannabis use and paranoia $(b=2.39$, BCa CI[.67, 4.40]) with a small to medium effect size (completely standardized indirect effect=.08; Sobel test $p<.01)$ for the indirect effect. Cognitive fusion mediated the relationship between cannabis use and psychotic-type experience ( $b=.62, \mathrm{BCa} \mathrm{CI}[.18,1.14])$ with a small to medium effect size (completely standardized indirect effect=.08; Sobel test $p<.01$ ) for the indirect effect. Cognitive fusion also mediated the relationship between cannabis use and distress $(b=2.15, \mathrm{BCa} \mathrm{CI}[.66,3.97])$ with a small to medium effect size (completely standardized indirect effect=.09; Sobel test $p<.01$ ) for the indirect effect.

\footnotetext{
${ }^{1}$ Summary table of indirect effects available on request.
} 
Cannabis-related paranoia

Study 2: In cannabis users, do external attribution and cognitive fusion account for the relationship between established risk factors and paranoia, psychotic-type experiences and distress?

\section{Correlations}

Table 2 gives descriptive statistics and Pearson's correlations for the cannabis using sub-sample. We found positive correlations between paranoia, psychotic-type experiences, distress, external attribution, cognitive fusion and schizotypy.

*Table 2 about here*

Analyses of variance

The MANOVA showed there was no impact of gender on paranoia, $F=.59$, partial $\eta^{2}=.01, p>.05$, psychotic-type experiences, $F=.75$, partial $\eta^{2}=.01, p>.05$, or distress, $F=.75$, partial $\eta^{2}=.01, p>.05$. There was also no impact of age of first cannabis-use on paranoia, $F=.0$, partial $\eta^{2}=.0, p>.05$, psychotictype experiences, $F=.39$, partial $\eta^{2}=.0, p>.05$, or distress, $F=.7$, partial $\eta^{2}=.0, p>.05$.

CSA was reported by $37.8 \%(n=65)$ of the cannabis-using sample, and this predicted paranoia, $F=19.96$, partial $\eta^{2}=.11, p<.001$, psychotic-type experiences, $F=9.51$, partial $\eta^{2}=.06,<.01$, and distress, $F=11.19$, partial $\eta^{2}=.06, p<.001$. A MANCOVA was used to examine the impact of CSA with external attribution and cognitive fusion as co-variates. When external attribution was added along with CSA, external attribution predicted paranoia, $F=19.50$, partial $\eta^{2}=.11, p<.001$, psychotic-type experiences, $F=66.17$, partial $\eta^{2}=.29, p<.001$, and distress, $F=39.36$, partial $\eta^{2}=.19, p<.001$. CSA remained a significant predictor of paranoia, $F=13.26$, partial $\eta^{2}=.07, p<.001$, and distress, $F=6.43, p<.05$, partial $\eta^{2}=.04$, but with reduced effect sizes. CSA was no longer related to psychotic-type experiences, $F=3.04, \eta^{2}=.02, p>.05$. When cognitive fusion was added, this predicted paranoia, $F=47.31$, partial $\eta^{2}=.22, p<.001$, psychotic-type experiences, $F=37.76$, partial $\eta^{2}=.19, p<.001$ and distress, $F=55.02$, 
Cannabis-related paranoia

partial $\eta^{2}=.25, p<.001$. CSA remained a significant predictor of paranoia, $F=7.21$, partial $\eta^{2}=.04, p<.01$ but with a reduced effect size. CSA was no longer related to psychotic-type experiences, $F=1.91$, partial $\eta^{2}=.01, p>.05$, or distress, $F=.297, p>.05$.

\section{Mediation analyses}

In the cannabis using sub-sample, external attribution did not mediate the relationship between schizotypy and paranoia ( $b=.02, \mathrm{BCa} \mathrm{CI}[-.001, .06])$ (completely standardized indirect effect=.04; Sobel test $p=.07)$. Cognitive fusion did mediate this relationship ( $b=.05, \mathrm{BCa}$ CI[.002,.104]) with a medium effect size (completely standardized indirect effect=.08; Sobel test $p<.05$ ) for the indirect effect. External attribution mediated the relationship between schizotypy and psychotic-type experiences $(b=.01, \mathrm{BCa} \mathrm{CI}[.004, .01])$ with a medium to large effect size (completely standardized indirect effect=.14; Sobel test $p<.01$ ) for the indirect effect. Cognitive fusion also mediated the relationship between schizotypy and psychotic-type experiences $(b=.02, \mathrm{BCa} \mathrm{CI}[.01, .04])$ with a medium to large effect size (completely standardized indirect effect=.13; Sobel test $p<.01$ ) for the indirect effect. Finally, external attribution mediated the relationship between schizotypy and distress $(b=.06, \mathrm{BCa} C I[.03, .11])$ with a medium effect size (completely standardized indirect effect=.10; Sobel test $p<.01$ ) for the indirect effect. Cognitive fusion also mediated the relationship between schizotypy and distress $(b=.01, \mathrm{BCa} \mathrm{CI}[.01, .19])$ with a medium to large effect size (completely standardized indirect effect=.19; Sobel test $p<.01$ ) for the indirect effect.

Study 3: Do people with psychosis and a cannabis-use history have higher levels of paranoia, psychotic experiences and distress, and is this accounted for by external attribution and cognitive fusion?

Inter-correlations are presented in Table 3. 
*Table 3 about here*

The MANOVA comparing people with psychosis with and without a cannabis-use history showed no difference between groups in paranoia, $F=3.83$, partial $\eta^{2}=.07, p>.05$, or distress, $F=1.43$, partial $\eta^{2}=.03, p>.05$. However, those with a history of cannabis use reported higher levels of psychotic experiences, $F=5.75$, partial $\eta^{2}=.10, p<.05$. A univariate analysis with psychotic experiences as the dependent variable and cannabis-use history as the independent variable, controlling for external attribution, found external attribution was a significant predictor of psychotic experiences, $F=67.30$, partial $\eta^{2}=.56, p<.001$, with cannabis-use history no longer significant, $F=.037$, partial $\eta^{2}=.0, p>.05$, partial $\eta^{2}=.0, p>.05$. A univariate analysis with psychotic experiences as the dependent variable and cannabis-use history as the independent variable, controlling for cognitive fusion, found cognitive fusion was significant for psychotic experiences, $F=14.28$, partial $\eta^{2}=21 ., p<.001$. Cannabis use history remained significant for psychotic experiences but with a reduced effect size, $F=4.11$, partial $\eta^{2}=.07 . p<.05$.

Study 3: In people with psychosis with a cannabis-use history, are risk factors associated with paranoia, psychotic experiences and distress, and are these relationships accounted for by external attribution and cognitive fusion?

There was no impact of gender on paranoia, $F=3.17$, partial $\eta^{2}=.06, p>.05$, or psychotic experiences, $F=3.50$, partial $\eta^{2}=.06, p>.05$. There was a significant impact of gender on distress, $F=4.69$, partial $\eta^{2}=.08, p<.05$, with higher scores for male participants. A univariate analysis was conducted with distress as the dependent variable and gender as the independent variable, controlling for external attribution. This was significant for external attribution $F=47.13$, partial $\eta^{2}=.46, p<.001$, but no longer 
significant for gender, $F=2.46$, partial $\eta^{2}=.04, p>.05$. A univariate analysis for distress as the dependent variable and gender as the independent variable, controlling for cognitive fusion was significant for cognitive fusion, $F=34.06$, partial $\eta^{2}=.38, p<.001$, and remained significant for gender, $F=7.2$, partial $\eta^{2}=.12, p<.05$.

The prevalence of CSA was higher in those with a cannabis-use history $(n=22 ; 59.5 \%)$ compared with no past cannabis use $(13.6 \%, n=3), \chi^{2}=11.9, p<.001$. Those with a sexual abuse history reported higher levels of paranoia, $F=9.86$, partial $\eta^{2}=.16, p<.01$, and psychotic experiences, $F=8.45$, partial $\eta^{2}=.14, p<.01$, but no difference in distress, $F=3.93$, partial $\eta^{2}=.07, p>.05$. A multivariate analysis with paranoia and psychotic experiences as the dependent variables, CSA as the independent variable, and external attribution as the covariate, showed that external attribution predicted greater paranoia, $F=13.51$, partial $\eta^{2}=.21, p<.01$, and psychotic experiences, $F=66.62$, partial $\eta^{2}=.56, p<.001$, with CSA no longer predictive of either paranoia, $F=2.82$, partial $\eta^{2}=.05, p>.05$, or psychotic experiences, $F=.39$, partial $\eta^{2}=.0, p>.05$. When cognitive fusion was added as a covariate, cognitive fusion predicted greater paranoia, $F=15.69$, partial $\eta^{2}=.23, p<.001$, and psychotic experiences, $F=11.0$, partial $\eta^{2}=.18, p<.01$. CSA remain a significant predictor but with a reduced effect size for paranoia, $F=5.55$, partial $\eta^{2}=.10, p<.05$, and psychotic experiences, $F=4.68$, partial $\eta^{2}=.08, p<.05$.

There was no impact of age of first cannabis-use on paranoia, $F=1.05$, partial $\eta^{2=} .03, p>.05$, or psychotic experiences, $F=2.34$, partial $\eta^{2}=.07, p>.05$. However, those who began using before 16 years reported higher levels of distress, $F=5.37$, partial $\eta^{2=} .14, p<.05$. A univariate analysis with distress as the dependent variable and age of first use as the independent variable, controlling for external attribution, was significant for external attribution, $F=33.7$, partial $\eta^{2}=.49, p<.05$. Age of first use was no longer significant, $F=2.29$, partial $\eta^{2}=.06, p>.05$. A univariate analysis with distress as the dependent variable and age of first use as the independent variable, controlling for cognitive fusion, was significant for cognitive fusion, $F=16.9$, partial $\eta^{2}=.33, p<.001$. Age of first use remained significant but with a reduced effect size: $F=4.34$, partial $\eta^{2}=.11, p<.05$. 
Cannabis-related paranoia

Schizotypy (high/low) predicted higher levels of paranoia, $F=41.32$, partial $\eta^{2}=.44, p<.001$, psychotic experiences, $F=28.45$, partial $\eta^{2}=.35, p<.001$ and distress, $F=31.27$, partial $\eta^{2}=.37, p<.001$. A multivariate analysis with paranoia, psychotic experiences and distress as the dependent variables, schizotypy as the independent variable, and external attribution as the co-variate, showed that external attribution predicted paranoia, $F=8.01$, partial $\eta^{2}=.13, p<.01$, psychotic experiences, $F=41.75$, partial $\eta^{2}=.45, p<.001$, and distress, $F=21.40$, partial $\eta^{2}=.29, p<.001$. Schizoptypy remained significant for paranoia, $F=17.04$, partial $\eta^{2}=.25, p<.001$, psychotic experiences, $F=5.44$, partial $\eta^{2}=.10 .0, p<.05$, and distress, $F=8.54$, partial $\eta^{2}=.14, p<.01$. A MANOVA was then conducted to examine the impact of schizotypy with cognitive fusion as a co-variate. Cognitive fusion was not significant for paranoia, $F=3.94$, partial $\eta^{2}=.07, p>.05$, or psychotic experiences, $F=1.55$, partial $\eta^{2}=.03, p>.05$, but was significant for distress $F=6.13$, partial $\eta^{2}=.11, p<.05$. Schizoptypy continued to predict greater paranoia, $F=13.65$, partial $\eta^{2}=.21, p<.01$, psychotic experiences, $F=10.49$, partial $\eta^{2}=.17, p<.01$ and distress, $F=7.89$, partial $\eta^{2}=.13, p<.01$.

Study 3: In people with psychosis, is using cannabis to manage negative affect associated with paranoia, psychotic experiences and distress, and is this accounted for by external attribution and cognitive fusion?

A MANOVA examined the impact of using cannabis to manage negative affect. Those who reported using cannabis in this way reported higher levels of paranoia, $F=6.13$ partial $\eta^{2}=.16, p<.05$, psychotic experiences, $F=6.79$, partial $\eta^{2}=.18, p<.05$, and distress, $F=6.79$, partial $\eta^{2}=.18, p<.05$. The MANOVA was repeated with external attribution as a co-variate. External attribution was significant for paranoia, $F=6.42$, partial $\eta^{2}=.17, p<.05$, psychotic experiences, $F=34.50$, partial $\eta^{2}=.53, p<.001$ and distress, $F=15.15$, partial $\eta^{2}=.45, p<.001$. Using cannabis to manage negative affect was no longer significant for paranoia, $F=2.18$, partial $\eta^{2}=.07, p>.05$, psychotic experiences, $\mathrm{F}=.1 .31$, partial $\eta^{2}=.04, p>.05$, or distress, $F=1.22$, partial $\eta^{2}=.04, p>.05$. The MANOVA was then repeated with cognitive fusion as a 
Cannabis-related paranoia

co-variate. Cognitive fusion was significant for paranoia, $F=5.43$, partial $\eta^{2}=.15, p<.05$, and distress, $F=8.08$, partial $\eta^{2}=.21, p<.01$, but not psychotic experiences, $F=1.85$, partial $\eta^{2}=.06, p>.05$. Using cannabis to manage negative affect was no longer significant for paranoia, $F=1.89$, partial $\eta^{2}=.06$, $p>.05$, psychotic experiences, $F=3.22$, partial $\eta^{2}=.09, p>.05$ or distress $F=1.62$ partial $\eta^{2}=.05, p>.05$.

\section{Discussion}

\section{Overview of findings}

In three linked studies we examined candidate mechanisms for the maintenance of cannabis-related psychosis. In a general population sample, cognitive fusion mediated the relationship between cannabis use and each of paranoia, psychotic-type experiences and distress. External attribution did not mediate these relationships.

In general population cannabis-users, contrary to expectation and previous research (Hamilton, et al., 2015; Valmaggia, et al., 2014), there was no impact of gender or age of first use on paranoia, psychotic-type experiences or linked distress. However, CSA predicted greater levels of paranoia, psychotic-type experiences and distress, consistent with evidence that people who have been abused in childhood are at greater risk of psychosis from cannabis-use (Harley et al., 2010; Konings et al., 2012; Shevlin et al., 2009). External attribution accounted for the relationship between CSA and psychotic-type experiences, and cognitive fusion accounted for the relationship between CSA and both psychotic-type experiences and distress. In this cannabis using sub-sample, external attribution mediated the relationships between schizotypy and both psychotic-type experiences and distress, with a non-significant trend for paranoia. Cognitive fusion mediated the relationships between schizotypy and each of paranoia, psychotic-type experiences and distress.

In people with psychosis, those with a history of cannabis-use showed higher levels of psychotic experiences compared with non-users, with external attribution and cognitive fusion fully or partially accounting for this relationship. In terms of established risk factors, male gender predicted 
Cannabis-related paranoia

greater distress, in line with previous findings (Hamilton et al., 2015), which was also fully or partially accounted for by external attribution and cognitive fusion.

People with psychosis and a cannabis-use history were more likely to have experienced CSA, and this was associated with higher levels of paranoia and psychotic experiences more broadly. Those who started using cannabis earlier reported higher levels of distress, and schizotypy was associated with higher levels of paranoia, psychotic experiences and linked distress. The results are consistent with evidence for the impact of these risk factors (Day et al., 2014; Spriggens \& Hides, 2015; Valmaggia et al., 2014), and suggests that these factors may increase psychosis in the clinical population via external attribution and cognitive fusion.

Finally, we examined the role of cannabis as a means of self-medicating, given the evidence that some people use cannabis to cope with paranoia and auditory hallucinations (Green et al., 2004; Schofield et al., 2006). Using cannabis to manage negative affect was associated with higher levels of paranoia, psychotic symptoms and linked distress. Again, these relationships were largely accounted for by external attribution and cognitive fusion.

\section{Limitations}

The findings are limited by our use of cross-sectional designs. While cross-sectional mediation is acceptable as an initial step in identifying potential mechanisms in established relationships (Disabato, 2016), longitudinal research is now needed to support a causal argument for the role of external attribution and cognitive fusion. Though sufficient for our main analyses, the clinical sample size did not allow for regression. Further limitations include the recruitment of convenience samples (the majority of whom were Caucasian), use of self-report questionnaires, and the fact that we did not screen Study 1 and 2 participants for psychotic disorders. 
Cannabis-related paranoia

\section{Initial model proposal}

Notwithstanding these limitations, the results suggest that external attribution and cognitive fusion play a key role in the development and maintenance of psychosis, and paranoia specifically, in people with a cannabis-use history. We present a model of cannabis-related paranoia (Figure 1) based on the model of social phobia (Clark \& Wells, 1995) and the model of paranoia (Stopa, Denton, Wingfield \& Newman-Taylor, 2013) derived from this. In the context of key risk factors, cannabis-use initiates affective, cognitive, behavioural and physiological responses, driven by the "processing of the self as an object of interpersonal threat" - the felt sense of paranoia in which internal events are experienced as necessarily accurate reflections of the self or reality. External attribution and cognitive fusion are highlighted as key psychological processes maintaining distressing paranoia.

*Figure 1 about here*

Male gender, CSA, schizotypy and younger age of first cannabis-use increase likelihood of transient psychotic-type experiences such as hearing voices, suspiciousness and unusual physical sensations from cannabis-use. These experiences are then more likely to be attributed externally and in a threatening manner (e.g. "they're going to get me") given the person's early learning (e.g. "others are dangerous"), rather than simply being attributed to cannabis intoxication. High levels of schizotypy also predispose unusual or supernatural explanations for anomalous experiences. An illustrative example is given in Figure 2.

*Figure 2 about here* 
Cannabis-related paranoia

\section{Conclusion}

This is the first study to demonstrate the potential role for external attribution and cognitive fusion in paranoia, psychotic-type experiences more broadly and linked distress, in general population, cannabis-using and clinical groups. These processes are highlighted in a proposed model which can be used as the basis for formulation driven therapy incorporating interventions which target external attribution and cognitive fusion to reduce risk of psychosis from cannabis use.

\section{Acknowledgments}

Thanks to all who took part, the expert by experience advisor (who wished to remain anonymous) and Shalini Raman. 
Cannabis-related paranoia

\section{References}

Bach, P., Hayes, S.C., \& Gallop, R. (2012). Long-term effects of brief acceptance and commitment therapy for psychosis. Behavior Modification, 36(2), 165-181.

Barkus, E., \& Lewis, S. (2008). Schizotypy and psychosis-like experiences from recreational cannabis in a non-clinical sample. Psychological Medicine, 38(9), 1267-1276.

Bernstein, A., Hadash, Y., Lichtash, Y., Tanay, G., Shepherd, K., \& Fresco, D.M. (2015). Decentering and related constructs. Perspectives on Psychological Science, 10(5), 599-617.

Bolderston, H., Gillanders, D.T., Turner, G., Taylor, H.C., Mhaoileoin, D., \& Coleman, A. (2019). The initial validation of a state version of the Cognitive Fusion Questionnaire. Journal of Contextual Behavioral Science, 12, 207-215.

Colizzi, M., \& Murray, R. (2018). Cannabis and psychosis: what do we know and what should we do? British Journal of Psychiatry, 212(4), 195-196.

Davidson, C.A., Hoffman, L., \& Spaulding, W.D. (2016). Schizotypal personality questionnaire: An update of norms, factor structure, and item content in a large non-clinical young adult sample. Psychiatry Research, 238, 345-355.

Day, N., Goldschmidt, L., Day, R., Larkby, C., \& Richardson, G. (2014). Prenatal marijuana exposure, age of marijuana initiation, and the development of psychotic symptoms in young adults. Psychological Medicine, 1-9.

Disabato, D. (2016). The double standard against cross-sectional mediation. Retrieved from http://www.daviddisabato.com/blog/2016/5/22/the-double-standard-against-cross-sectionalmediation

Fenigstein, A., \& Vanable, P.A. (1992). Paranoia and self-consciousness. Journal of Personality and Social Psychology, 62(1), 129. 
Cannabis-related paranoia

Ferdinand, R., Sondeijker, F., van der Ende, J., Selten, J., Huizink, A., \& Verhulst, F. C. (2005). Cannabis use predicts future psychotic symptoms, and vice versa. Addiction, 100(5), 612-618.

Freeman, D., Dunn, G., Murray, R.M., Evans, N., Lister, R., Antley, A., ... Williams, J. (2014). How cannabis causes paranoia: using the intravenous administration of $\Delta$ 9-tetrahydrocannabinol (THC) to identify key cognitive mechanisms leading to paranoia. Schizophrenia Bulletin, 41(2), 391-399.

Fridberg, D., Vollmer, J., O'Donnell, B., \& Skosnik, P. (2011). Cannabis users differ from non-users on measures of personality and schizotypy. Psychiatry Research, 186(1), 46-52.

Gage, S.H., Hickman, M., \& Zammit, S. (2016). Association between cannabis and psychosis: epidemiologic evidence. Biological Psychiatry, 79(7), 549-556.

Gillanders, D.T., Bolderston, H., Bond, F.W., Dempster, M., Flaxman, P.E., Campbell, L., ... Ferenbach, C. (2014). The development and initial validation of the Cognitive Fusion Questionnaire. Behavior Therapy, 45(1), 83-101.

Green, B., Kavanagh, D., \& Young, R.M. (2004). Reasons for cannabis use in men with and without psychosis. Drug and Alcohol Review, 23(4), 445-453.

Gregg, L., Barrowclough, C., \& Haddock, G. (2007). Reasons for increased substance use in psychosis. Clinical Psychology Review, 27(4), 494-510.

Griffith-Lendering, M.F.H., Wigman, J.T.W., Prince van Leeuwen, A., Huijbregts, S.C.J., Huizink, A.C., Ormel, J., ... Vollebergh, W.A.M. (2013). Cannabis use and vulnerability for psychosis in early adolescence — a TRAILS study. Addiction, 108(4), 733-740.

Hamilton, I., Galdas, P., \& Essex, H. (2015). Cannabis psychosis, gender matters. Advances in Dual Diagnosis, 8(3), 153-162.

Hayes, A.F. (2017). The PROCESS macro for SPSS and SAS. 
Cannabis-related paranoia

Horan, W.P., Blanchard, J.J., Clark, L.A., \& Green, M.F. (2008). Affective traits in schizophrenia and schizotypy. Schizophrenia Bulletin, 34(5), 856-874.

Houston, J.E., Murphy, J., Shevlin, M., \& Adamson, G. (2011). Cannabis use and psychosis: re-visiting the role of childhood trauma. Psychological Medicine, 41(11), 2339-2348.

Khantzian, E. J. (1997). The self-medication hypothesis of substance use disorders: A reconsideration and recent applications. Harvard Review of Psychiatry, 4(5), 231-244.

Kinderman, P., \& Bentall, R.P. (1996). A new measure of causal locus: the internal, personal and situational attributions questionnaire. Personality and Individual Differences, 20(2), 261-264.

Kolliakou, A., Joseph, C., Ismail, K., Atakan, Z., \& Murray, R. M. (2011). Why do patients with psychosis use cannabis and are they ready to change their use? International Journal of Developmental Neuroscience, 29(3), 335-346.

Ksir, C., \& Hart, C.L. (2016). Cannabis and psychosis: a critical overview of the relationship. Current Psychiatry Reports, 18(2), 12.

Lembke, A. (2012). Time to abandon the self-medication hypothesis in patients with psychiatric disorders. The American Journal of Drug and Alcohol Abuse, 38(6), 524-529.

Loewy, R.L., Pearson, R., Vinogradov, S., Bearden, C.E., \& Cannon, T.D. (2011). Psychosis risk screening with the Prodromal Questionnaire-brief version (PQ-B). Schizophrenia Research, 129(1), 42-46.

Mané, A., Fernández-Expósito, M., Bergé, D., Gómez-Pérez, L., Sabaté, A., Toll, A., ... \& Perez, V. (2015). Relationship between cannabis and psychosis: reasons for use and associated clinical variables. Psychiatry Research, 229(1-2), 70-74. 
Cannabis-related paranoia

Manrique-Garcia, E., Zammit, S., Dalman, C., Hemmingsson, T., Andreasson, S., \& Allebeck, P. (2014). Prognosis of schizophrenia in persons with and without a history of cannabis use. Psychological Medicine, 44(12), 2513-2521.

Marconi, A., Di Forti, M., Lewis, C.M., Murray, R.M., \& Vassos, E. (2016). Meta-analysis of the association between the level of cannabis use and risk of psychosis. Schizophrenia Bulletin, 42(5), 1262-1269.

Mason, O., Morgan, C.J.A., Dhiman, S.K., Patel, A., Parti, N., Patel, A., \& Curran, H.V. (2009). Acute cannabis use causes increased psychotomimetic experiences in individuals prone to psychosis. Psychological Medicine, 39(6), 951-956.

McHugh, M.J., McGorry, P.D., Yung, A.R., Lin, A., Wood, S.J., Hartmann, J.A., \& Nelson, B. (2017). Cannabis-induced attenuated psychotic symptoms: implications for prognosis in young people at ultra-high risk for psychosis. Psychological Medicine, 47(4), 616-626.

Murray, R. M., Quigley, H., Quattrone, D., Englund, A., \& Di Forti, M. (2016). Traditional marijuana, high-potency cannabis and synthetic cannabinoids: increasing risk for psychosis. World Psychiatry, 15(3), 195-204.

Mustonen, A., Niemelä, S., Nordström, T., Murray, G.K., Mäki, P., Jääskeläinen, E., \& Miettunen, J. (2018). Adolescent cannabis use, baseline prodromal symptoms and the risk of psychosis. British Journal of Psychiatry, 212(4), 227-233.

Radhakrishnan, R., Wilkinson, S. T., \& D’Souza, D. C. (2014). Gone to pot - A review of the association between cannabis and psychosis. Frontiers in Psychiatry, 5, 54.

Richardson, T., Hughes, M., Leech, K., \& Raman, S. (2014). Letter to the Editor: Cannabis use and transition to psychosis: is interpretation of unpleasant effects a mediating variable? Psychological Medicine, 1-1. 
Cannabis-related paranoia

Schoeler, T., Monk, A., Sami, M.B., Klamerus, E., Foglia, E., Brown, R., ... \& Bhattacharyya, S. (2016). Continued versus discontinued cannabis use in patients with psychosis: a systematic review and meta-analysis. The Lancet Psychiatry, 3(3), 215-225.

Shevlin, M., Murphy, J., Houston, J.E., \& Adamson, G. (2009). Childhood sexual abuse, early cannabis use, and psychosis: Testing the effects of different temporal orderings based on the National Comorbidity Survey. Psychosis, 1(1), 19-28.

Spriggens, L., \& Hides, L. (2015). Patterns of cannabis use, psychotic-like experiences and personality styles in young cannabis users. Schizophrenia Research, 165(1), 3-8.

Stopa, L., Denton, R., Wingfield, M., \& Newman-Taylor, K. (2013). The fear of others: a qualitative analysis of interpersonal threat in social phobia and paranoia. Behavioural and cognitive psychotherapy, 41(2), 188-209.

Strosahl, K.D., Hayes, S.C., Wilson, K.G., \& Gifford, E.V. (2004). An ACT primer. In A practical guide to acceptance and commitment therapy (pp.31-58). Springer, Boston, MA.

Teasdale, J.D., Moore, R.G., Hayhurst, H., Pope, M., Williams, M., \& Segal, Z.V. (2002). Metacognitive awareness and prevention of relapse in depression: empirical evidence. Journal of Consulting and Clinical Psychology, 70(2), 275.

Valmaggia, L.R., Day, F.L., Jones, C., Bissoli, S., Pugh, C., Hall, D., ... McGuire, P.K. (2014). Cannabis use and transition to psychosis in people at ultra-high risk. Psychological Medicine, 44(12), 2503-2512.

Van der Meer, F.J., Velthorst, E., \& Outcome of Psychosis (GROUP) Investigators. (2015). Course of cannabis use and clinical outcome in patients with non-affective psychosis: a 3-year follow-up study. Psychological Medicine, 45(9), 1977-1988. 